\title{
Review Article \\ Peritoneal Membrane Injury and Peritoneal Dialysis
}

\author{
Shaan Chugh, Sultan Chaudhry, Timothy Ryan, and Peter J. Margetts \\ Department of Medicine, McMaster University, Division of Nephrology, St. Joseph's Hospital, 50 Charlton Avenue E, Hamilton, ON, \\ Canada L8P $4 A 6$ \\ Correspondence should be addressed to Peter J. Margetts; margetts@mcmaster.ca
}

Received 6 July 2014; Revised 24 September 2014; Accepted 29 September 2014; Published 2 November 2014

Academic Editor: Lawrence H. Lash

Copyright (C) 2014 Shaan Chugh et al. This is an open access article distributed under the Creative Commons Attribution License, which permits unrestricted use, distribution, and reproduction in any medium, provided the original work is properly cited.

\begin{abstract}
For patients with chronic renal failure, peritoneal dialysis (PD) is a common, life sustaining form of renal replacement therapy that is used worldwide. Exposure to nonbiocompatible dialysate, inflammation, and uremia induces longitudinal changes in the peritoneal membrane. Application of molecular biology techniques has led to advances in our understanding of the mechanism of injury of the peritoneal membrane. This understanding will allow for the development of strategies to preserve the peritoneal membrane structure and function. This may decrease the occurrence of PD technique failure and improve patient outcomes of morbidity and mortality.
\end{abstract}

\section{Introduction}

PD involves both diffusive and convective clearance driven mainly by glucose-based hyperosmolar PD fluid. The peritoneal membrane overlies the surface of all intra-abdominal organs, the diaphragm, and the parietal peritoneal wall. The peritoneal membrane is a fairly simple structure, with a superficial epithelial-like cell layer-the mesotheliumwhich is attached to a basement membrane (Figure 1). Beneath the basement membrane is a submesothelial layer consisting of connective tissue, fibroblasts, and blood vessels. Under optimal conditions, the peritoneum acts as an efficient, semipermeable dialysis membrane, enabling removal of metabolites, uremic toxins, salt, and water from the patient.

The rate of removal of these products from the blood correlates with the vascular surface area in contact with PD fluids in the peritoneal cavity [1]. Peritoneal membrane solute transport is commonly quantified as a dialysate to plasma ratio of solute (i.e., $d / p$ creatinine). Increased peritoneal membrane solute transport should confer benefit for the patient as blood clearance would be more efficient. However, many studies have demonstrated the opposite [2]. A meta-analysis of observational studies demonstrated that every 0.1 increase in $\mathrm{d} / \mathrm{p}$ creatinine carries a $15 \%$ increased risk of mortality [3]. This risk may be modified by the use of nocturnal cycling PD and use of alternate fluids such as icodextrin [4]. The mechanism whereby increased peritoneal solute transport is associated with increased mortality has not been clearly elucidated. Increased peritoneal membrane solute transport leads to increased absorption of glucose from the PD fluid [5]. This causes a rapid loss of the ultrafiltration gradient with decreased ultrafiltration, chronic volume expansion [6], hypertension [7], and adverse cardiovascular outcomes [8]. Furthermore, the increased glucose absorption may decrease food intake and lead to malnutrition [4]. There may also be common mechanisms, such as inflammation, which underlie both the increased solute transport and the increased mortality [9].

Solute transport is associated with peritoneal vascular surface area and has been modeled using the "three-pore" concept from Rippe and colleagues [10]. The "three-pore" model assumes the peritoneal membrane is a two-dimensional structure and the main barrier to solute and water transport is the endothelial cell layer of the blood vessels. The "three pores" refers to 3 different structures-aquaporin, small, and large pores-within the endothelial cell layer, which are size selective in restricting solute transport. Aquaporin-1 composes the smallest pore in the three-pore model. These channels allow for water transport by way of the crystalloid osmotic gradient. Small pores do the majority of the work in PD and mediate the transport of low molecular weight solutes. Large pores allow for the passage of proteins with higher molecular weight such as albumin, transferrin, and $\operatorname{IgG}[10]$. 


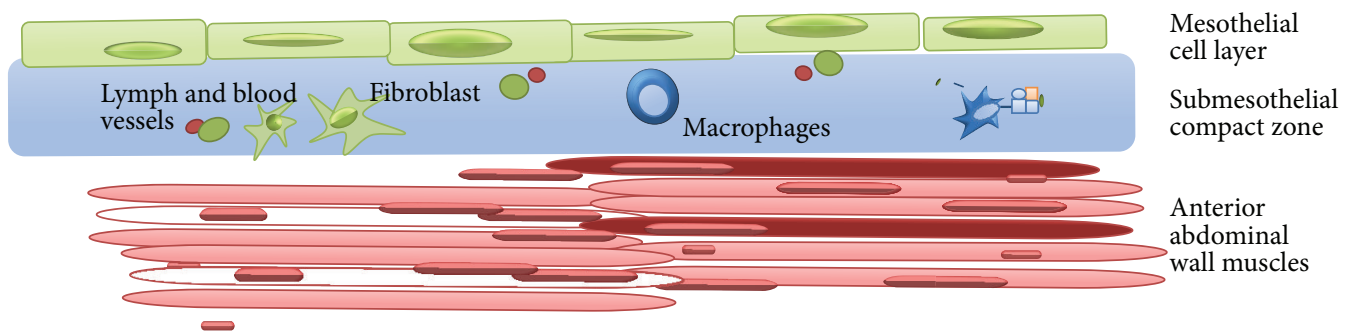

(a)

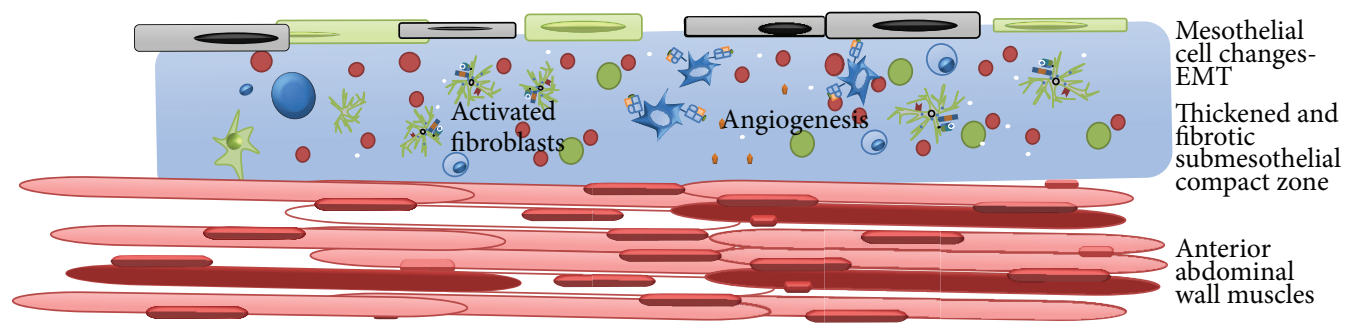

(b)

FIGURE 1: Changes in the peritoneal membrane with dialysis treatment. (a) Normal peritoneal membrane consists of an intact mesothelium overlying a thin submesothelial compact zone containing extracellular matrix, blood vessels, and a few scattered cells-fibroblasts and peritoneal macrophages. (b) After time on dialysis, activated fibroblasts or myofibroblasts appear along with increased submesothelial extracellular matrix and angiogenesis. Mesothelial cells are injured and sometimes denuded from the peritoneal surface.

Ultrafiltration is more complex and clinical modeling data suggests that both angiogenesis and increase in extracellular matrix (i.e., fibrosis) are required for ultrafiltration dysfunction [11]. Neovascularization of peritoneal tissue also has implications for ultrafiltration. Increasing vascular surface area causes increased loss of glucose from the peritoneal cavity effectively contributing to a reduction in the osmotic gradient as well [12]. These histologic changes are driven by clinical factors such as nonbiocompatible dialysate, glucose, uremia, peritonitis, and inflammation. One common downstream mediator of both peritoneal membrane fibrosis and angiogenesis appears to be transforming growth factor beta (TGFB) [13].

Therefore, ultrafiltration dysfunction is common and progresses over time on therapy and eventually leads to technique failure [14]. There is increasing evidence that both angiogenesis and expansion of the vascular surface along with peritoneal fibrosis are required for ultrafiltration failure to develop [11].

\section{Profiling of the Peritoneal Membrane over Time}

In a seminal study, Williams and colleagues studied peritoneal biopsies from $113 \mathrm{PD}$ patients [15]. They demonstrated that over time on dialysis there was a progressive increase in submesothelial thickening and a unique vasculopathy. The vasculopathy appeared as vessel wall sclerosis and luminal narrowing. The degree of vasculopathy correlated with time on PD treatment and with overall submesothelial fibrosis [16]. There was an increase in the number of blood vessels in the peritoneal tissues of patients on PD which was more pronounced in those with peritoneal membrane ultrafiltration failure [15]. These histologic changes are associated with changes in peritoneal membrane function. This has been elegantly demonstrated by Davies in observations from a large PD patient cohort followed over time [17]. These changes include a progressive increase in solute transport measured by $\mathrm{d} / \mathrm{p}$ creatinine and an associated decrease in ultrafiltration capacity [17].

There is a progression in the histologic changes seen in the peritoneal membrane with time on dialysis. Early on, changes in the mesothelium manifest as microvilli loss and signs of mesothelial injury such as cellular hypertrophy and increased vacuolation. Eventually, mesothelial cells detach from their basement membrane [18]. Over time, the presence of visceral and parietal simple sclerosis becomes evident and is quite common in patients who have been on long-term PD [19]. Mesothelial cell denudation as well as acellular sceloritic changes within the submesothelial connective tissue may also occur in conjunction with peritoneal sclerosis. A different and more rare form of peritoneal fibrosis, encapsulating peritoneal sclerosis (EPS), can occur and have fatal outcomes. Aberrations at the cellular and molecular level that are characteristic of EPS include fibrin deposition, fibroblast activation, and capillary angiogenesis [20]. The role of inflammation in EPS has been described [18].

\section{Epithelial to Mesenchymal Transition}

Central to the progression of peritoneal fibrosis and angiogenesis are changes in the epithelial-like mesothelial cell layer that lines the peritoneal cavity. Injury to the peritoneal tissues induces transition of the mesothelial cells to a 
mesenchymal phenotype-a phenomenon referred to as epithelial mesenchymal transition (EMT). This phenomenon has been described in various biological settings including organogenesis [21], metastatic transformation of cancer [22], and fibrosis [23]. EMT involves downregulation of epithelial markers such as the intercellular adhesion molecule ECadherin, upregulation of mesenchymal markers such as alpha-smooth muscle actin (a-sma), cytoskeletal rearrangement leading to increased cellular motility, and invasion into the interstitial tissue, usually across a basement membrane barrier. The injured epithelial cell transitions into a submesothelial myofibroblast [24]. Myofibroblasts are specialized extracellular matrix secreting cells with contractile properties that are highly associated with fibrotic tissue [25].

In the setting of peritoneal fibrosis, EMT has been experimentally induced by a number of agents associated with PD including high glucose concentration [26], glucose degradation products (GDP) [27], and peritoneal inflammation [28]. We have identified TGFB as a direct mediator of EMT in the peritoneum [29]. TGFB is a member of a large family of structurally related cytokines involved in growth and differentiation that includes activins and bone morphogenic proteins (BMP). Epithelial transition appears to occur when a balance between pro-EMT growth factors, such as TGFB, overbalances the protective factors such as BMP7. Peritoneal EMT can be reversed and the peritoneal membrane preserved by overexpressing the protective BMP7 [30].

The members of the TGFB superfamily signal through common receptors and utilize common signaling molecules. The canonical signaling pathway involves the SMAD proteins. We have further dissected this pathway by examining the role of SMAD3 in TGFB induced peritoneal fibrosis [31]. We found that in $\mathrm{SMAD}^{-1-}$ mice, TGFB did not induce fibrosis or angiogenesis. There was, however, persistence of TGFB induced EMT that was abrogated by blockade of the mammalian target of rapamycin (mTOR) pathway. Although the SMAD signaling pathway is the dominant pathway involved in response to TGFB, multiple other signaling pathways are also activated in the setting of TGFB induced fibrosis. We demonstrated in vivo that TGFB induced betacatenin signaling and this effect was inhibited by rapamycin [31].

Further down the signaling pathway, the EMT program appears to be controlled by a group of transcription factors including Snaill, Snail2 (Slug), and Twist [32]. These factors regulate expression of genes involved in the EMT process such as E-Cadherin and the matrix metalloproteinases (MMP) [33].

Although we have provided substantial evidence for TGFB mediated peritoneal EMT using standard dual labeling studies along with electron microscopy [29], and this has been supported by studies from other groups using different stimuli $[26,30,34,35]$, EMT as a prime mechanism of fibrosis has come under question recently. For example, EMT of renal tubular epithelial cells was once felt to be a major source of interstitial myofibroblasts and renal fibrosis [36]. Careful studies using cell lineage marking has shown that the pericyte appears to be the origin of interstitial myofibroblast leading to fibrosis [37]. More recently, by tracking the fate of mesothelial cells using cell specific promoters, Chen and colleagues have demonstrated that transitioned mesothelial cells make up few, if any, of the interstitial myofibroblasts in the peritoneum [38]. These results are intriguing and suggest that we will need to rethink the role of the mesothelium in peritoneal membrane injury and fibrosis. These cells are unlikely to be direct participants as transformed myofibroblasts, instead, they likely remain an essential component of peritoneal fibrosis by transmitting the injury signal in the peritoneal cavity to the submesothelial fibroblasts and vasculature in the form of fibrogenic and angiogenic cytokines.

Under certain circumstances, we have found that epithelial cells can undergo injury and transition to a mesenchymal phenotype without invasion into the submesothelial tissue. After adenovirus mediated overexpression of platelet derived growth factor (PDGF) B [39] or hypoxia inducible factor 1 alpha (HIFla) [40], we found clear evidence of cellular transition with dual labeling of mesothelial cells (cytokeratin) with myofibroblast markers (a-sma). Despite this, no dual labeled cells were observed in the submesothelial tissue. In the case of PDGF-B, we attributed this phenomenon to a lack of induction of MMPs, specifically MMP2 and MMP9, which we hypothesized were necessary to degrade the basement membrane to allow for mobilization of transitioned epithelial cells [39].

We have also demonstrated that transitioned epithelial cells are a source for vascular endothelial growth factor (VEGF) and thus promote peritoneal angiogenesis [41]. This is supported by evidence from Aroeira and colleagues from ex vivo peritoneal mesothelial cell cultures [42]. They showed that if cells from peritoneal effluent had a fibroblast as opposed to an epithelial phenotype in culture, this was associated with peritoneal membrane injury and EMT. These fibroblast-like cells were grown from patients with increased peritoneal membrane transport and these cells produced more VEGF than epithelial-like cells [42].

Therefore, although there is some controversy as to the role of EMT in establishing submesothelial myofibroblasts and peritoneal fibrosis, it is clear that mesothelial cells play a role in peritoneal membrane damage; they undergo cellular changes in response to injury and secrete various factors such as VEGF that are responsible for the histologic changes in the peritoneal tissues. Whether the peritoneal mesothelial cell is a direct or indirect agent in peritoneal membrane fibrosis and angiogenesis, protecting the mesothelium is arguably a logical therapeutic goal in preserving long-term peritoneal membrane function.

\section{A Central Role for TGFB in Peritoneal Membrane Injury}

TGFB is a growth factor that is central to the development of sis [43] and associated with the fibrotic and angiogenic responses observed in long-term PD patients (Figure 2). Published evidence from our group and others $[13,44]$ indicates that TGFB plays an essential role in peritoneal fibrosis and EMT. Glucose [45], GDPs [46], and inflammation [47] are linked to increased TGFB expression in mesothelial cells. 


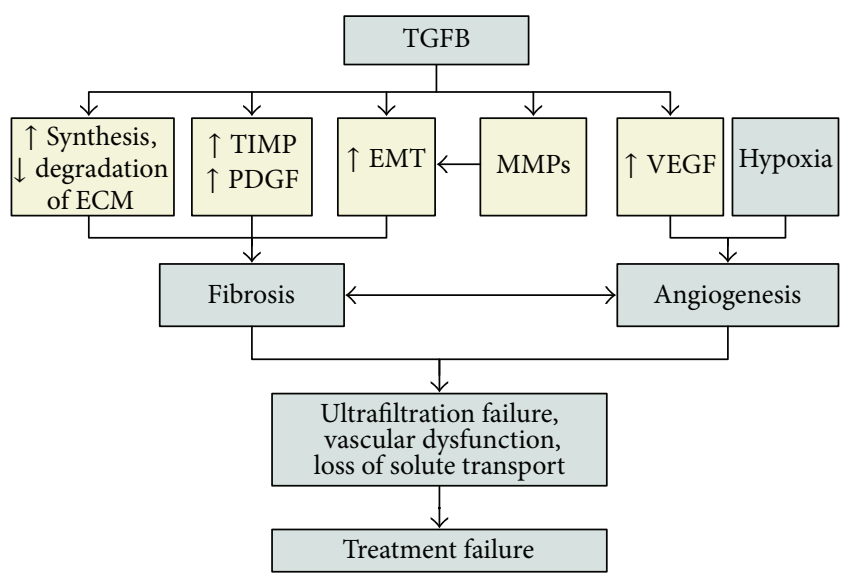

FIGURE 2: Role of transforming growth factor beta (TGFB) in peritoneal membrane injury. TGFB has multiple actions directly on elaboration of extracellular matrix (ECM), upregulation of various growth factors and metalloproteinases (MMPs), and induction of epithelial mesenchymal transition (EMT). TGFB also induces blood vessel growth directly through vascular endothelial growth factor (VEGF) and secondarily through hypoxia driven mechanisms.

In animal models, it has been shown that blocking TGFB signalling using SMAD7 [48, 49] or BMP7 [50] prevents mesothelial cell transition and peritoneal injury. Finally, there is increasing human data including our own observations [51], demonstrating an association between peritoneal effluent TGFB concentration to peritoneal solute transport [52].

We have shown that adenovirus-mediated gene transfer of TGFB1 to the peritoneum results in functional and structural changes similar to those seen in patients on longterm PD in both rats [13] and mice [31]. These changes include fibrosis, angiogenesis, increased solute transport, and ultrafiltration dysfunction. Furthermore, using a helper dependent adenovirus to deliver prolonged TGFB1 expression in mice, we observed peritoneal changes including bowel encapsulation and adhesion identical to that seen in PD patients with EPS [53].

The SMAD signaling pathway is integral to TGFB induced peritoneal fibrosis and angiogenesis. We have shown that these processes are abrogated in $\mathrm{SMAD}^{-/-}$mice exposed to and adenovirus expressing TGFB [31]. Other TGFB mediated pathways are likely involved. Peng and colleagues reduced peritoneal fibrosis and angiogenesis in rats on daily $\mathrm{PD}$ by using fasudil to inhibit the Rho/Rho associated protein kinase (ROCK) pathway [54]. Recently TGFB associated kinase-1 [55] and p38 [56] have been identified as TGFB regulated molecules important in peritoneal membrane injury and fibrosis.

\section{TGFB and the Role of Angiogenesis in Peritoneal Membrane Dysfunction}

Angiogenesis is a complex process involving initiation, progression, and maintenance of new vasculature arising from existing blood vessels. The association between vascularization of the peritoneal tissue and ultrafiltration dysfunction has been demonstrated in animal models [12, 57] and in human biopsy studies $[58,59]$. We have directly demonstrated the causative effect of peritoneal vascularization using adenovirus-delivered antiangiogenic therapy in an animal model of peritoneal membrane injury. We showed that an adenovirus expressing angiostatin reduced peritoneal vascularization and improved ultrafiltration function [12].

Several lines of evidence support a direct role of TGFB in angiogenesis. TGFB1 deficient mice have lethal defects in blood vessel maturation and hematopoiesis [60]. The TGFB receptor ALK-1 is involved in the signaling that leads to vascular maturation $[61,62]$. TGFB has been hypothesized to have a role in the maturation of VSMCs after their recruitment by PDGF [63]. TGFB synergistically acts with HIF la $[64,65]$ and high glucose [66] in upregulating VEGF and induces expression of angiopoietin-1, thus stabilizing blood vessels during fibrogenesis $[67,68]$.

TGFB is responsible for peritoneal angiogenesis through at least two mechanisms. As mentioned above, TGFB directly induces VEGF and angiogenesis [13]. This is best seen in mesothelial cells which undergo an EMT process in response to TGFB and become a source for VEGF [41, 42]. TGFB also induces an expansion of the submesothelial extracellular matrix. We have shown that this expanded submesothelial tissue becomes hypoxic, and this hypoxia drives a secondary angiogenic response [40]. Specifically, we found that TGFBinduced submesothelial tissue expressed HIFla which is a key regulator of the hypoxic response. Regulation of hypoxia is mainly at the posttranslational level [69]. However, several cytokines and signaling pathways have been demonstrated to increase gene expression of HIFla, most notably the PI3K/Akt pathway. This interaction occurs through mTOR and inhibition of this pathway with rapamycin downregulates HIFla gene expression [70]. We demonstrated that in the peritoneum, rapamycin did not block the direct TGFB induced angiogenesis but did prevent the secondary hypoxia driven angiogenic response [40].

We also demonstrated that HIFla overexpression alone could induce fibrosis and angiogenesis in the mouse peritoneum [40]. Therefore, fibrosis appears to induce a hypoxic response, but hypoxia can also induce fibrosis. Hypoxia has been shown to upregulate TGFB in human umbilical endothelial cell culture $[71,72]$. In cultured lung fibroblasts, hypoxia and TGFB were found to interact to alter the MMP/tissue inhibitor of metalloproteinase balance [73]. This balance is important in collagen metabolism and the establishment of a "profibrotic environment" $[47,74]$. Connective tissue growth factor, a cysteine rich protein strongly associated with fibrosis [75], has a hypoxia responsive element in the promoter region and is upregulated in cultured renal tubular cells exposed to low oxygen tension [76]. Higgins and colleagues demonstrated that HIFla could directly induce EMT and fibrosis in renal tubular epithelial cells [77].

\section{Risk Factors for Peritoneal Membrane Injury}

Commonly used PD solutions are characterized by acidic $\mathrm{pH}$, high glucose concentration, high lactate concentrations, high 


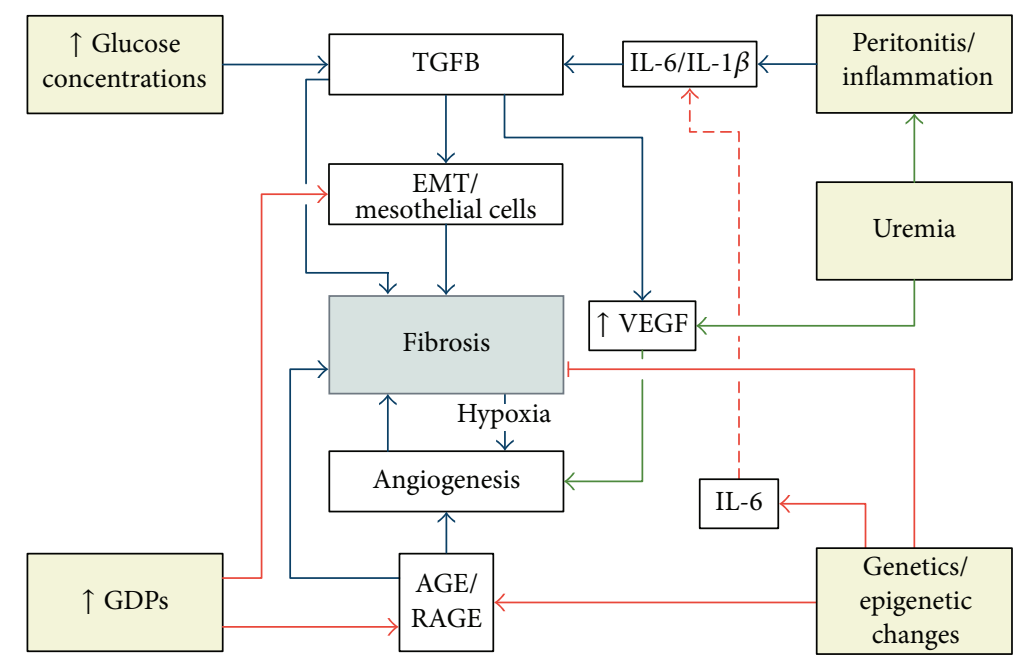

FIGURE 3: Mechanisms of peritoneal membrane injury. Factors related to PD solutions (increased glucose exposure and glucose degradation products (GDPs)) along with patient specific factors (peritonitis, inflammation, uremia, and genetic predisposition) are responsible for injury to the peritoneal membrane. Transforming growth factor beta (TGFB) is a central player in translating the injury signal to changes in the tissue. Inflammation is mediated by interleukin (IL) 6. GDPs induce advanced glycation end-products (AGEs) that can bind to the receptor for AGE (RAGE) to directly induce fibrosis. Angiogenesis is mediated by vascular endothelial growth factor (VEGF) and hypoxia.

overall osmolality, and GDPs which are a by-product of standard sterilization procedure. The demonstrated detrimental effect of these bioincompatible solutions on the peritoneal membrane has led to the development and testing of novel strategies and solutions in an attempt to preserve the peritoneal membrane [78]. The overall clinical impact of these more biocompatible solutions is not clear [79]. However, in addition to solution type, modifiable patient risk factors are equally important in patients undergoing PD (Figure 3).

(1) PD Fluid Related Factors. Long-term exposure of the peritoneal membrane to high glucose concentrations will cause changes in membrane permeability and structure. In vitrostudies by Kang and colleagues demonstrated that bioincompatible solutions containing high glucose concentrations will indeed stimulate the mesothelial cells to produce TGFB [80]. From a functional standpoint, patients exposed to hypertonic glucose dialysate demonstrate an earlier loss in residual renal function [81]. Moreover, high glucose concentrations have been recently linked with an increased mortality secondary to cardiovascular disease emphasizing the need for biocompatible solutions [82].

Glucose likely has a detrimental effect on the peritoneal membrane both from systemic hyperglycemia and local effects of dialysis fluid. Rodent studies suggests that streptozotocin induced diabetes causes increased peritoneal membrane solute transport, an effect that is mediated by VEGF [83]. In patients, the association between diabetes and solute transport at initiation of dialysis has been observed in some studies [84] but not others [85].

The heat sterilization process of PD fluids creates GDPs, which react with proteins to produce advanced glycation endproducts (AGE). Both GDPs and AGEs have been shown to have a detrimental effect on the peritoneal membrane, perhaps mediated through the receptor for AGE [86]. Newer solutions have been developed which drastically reduce the concentration of GDPs created during sterilization. These biocompatible solutions have shown some clinical promise in PD patients [87], with a suggestion of a beneficial effect on peritoneal membrane function [88]. Longer duration studies are likely required to definitively assess the true effect of these solutions.

(2) Patient Related Factors. Peritonitis is a well-recognized complication seen in patients treated with PD. Animal models have demonstrated that frequent peritonitis occurrence may cause increased circulating TGFB mRNA expression in peritoneal cells [89]. Subsequent research from our group showed that adenovirus mediated gene transfer of interleukin (IL) $1 \mathrm{~B}$ or tumor necrosis factor (TNF) mimicked inflammatory changes seen in peritonitis. Persisting fibrotic response, angiogenesis, and ultrafiltration dysfunction were related to overexpression of IL-1B, whereas TNF induced transient changes in the peritoneal membrane [90]. A single episode of peritonitis has been shown to have a detrimental effect on the peritoneal membrane as measured by changes in solute transport rates [91, 92].

End stage kidney disease is known to induce systemic inflammation. IL- 6 is a pleiotropic cytokine with a diverse range of function that was first isolated in 1986 [93]. IL-6 has been implicated in the pathogenesis of peritoneal inflammation in those undergoing PD and is associated with increased peritoneal solute transport [94]. Later, in the global fluid study of over 900 PD patients, Lambie and colleagues demonstrated that systemic inflammation, measured by serum IL6 , was associated with increased risk of mortality, whereas peritoneal inflammation was a distinct process and associated with increased peritoneal solute transport [95]. This cytokine, released following exposure to dialysate, has a role in the regulation of the switch from acute to chronic inflammation 
of the peritoneal membrane. IL- 6 also stimulates the downstream production of acute phase proteins such as angiogenic molecules, chemokines, and adhesion molecules [94].

Interestingly, the uremic state alone induces changes in the peritoneal membrane. This was best observed in peritoneal biopsy data where biopsies from uremic patients at the time of PD catheter insertion and before any exposure to dialysis fluid already showed significant submesothelial thickening [15]. The impact of uremia on the peritoneal membrane is supported by observations in rodents [96]. The presence of uremia in the background of diabetes has also showed to contribute to peritoneal thickening primarily through hyalinizing vasculopathy within capillaries [97]. These alterations become more apparent with PD and can affect transport.

(3) Genetics. There is increasing evidence to suggest that genetic variation plays a significant role in peritoneal membrane solute transport and peritoneal membrane fibrosis. There is evidence to support an association between peritoneal membrane solute transport and gene polymorphisms of IL-6 [98, 99], endothelial nitric oxide synthase [100, 101], and the receptor for AGE [102]. An association was not found between solute transport and VEGF [98, 102, 103], IL-10 [99, $104]$, and TNF $[99,104]$. An association was found between a RAGE gene polymorphism and the presence of EPS [105].

Recently, we evaluated the peritoneal fibrogenic response in 4 mice strains that span the genetic spectrum of inbred mice [106]. Strain dependence of the fibrogenic response has also been observed in models of kidney [107], liver [108] and heart [109] disease and supports the hypothesis that genetics play a role in the peritoneal membrane response to PD therapy.

(4) Epigenetics. A hallmark of fibrogenic changes is that the condition tends to progress even when the inciting stimulus is removed [110, 111]. This is specifically relevant for EPS; Balasubramaniam reported on a cohort of $111 \mathrm{PD}$ patients who developed EPS [112]. Fifty-one patients were diagnosed after the cessation of PD, with 21 being diagnosed after more than 3 months on hemodialysis. Additional 14 patients were diagnosed after a renal transplant. This suggests that the fibrogenic process, once initiated, is sustained despite removal of the inciting agent (PD therapy). One compelling explanation is that environmental triggers induce epigenetic changes in the resident cells (mesothelial cells or fibroblasts), and these "reprogrammed" cells take on a persisting fibrogenic phenotype. These "activated" fibroblasts have been observed in many disease processes involving fibrosis [111]. In a seminal paper, Bechtel and colleagues found that activated fibroblasts in a model of renal fibrosis demonstrated hypermethylation of the promoter region of the RASAL1 gene [113]. This hypermethylation decreased RASAL1 gene expression and allowed for persisting activity of the RAS pathway.

The epigenetic controls over gene expression primarily include methylation of cytosine residues and histone modifications. These processes have been observed in diseases associated with fibrosis [114]. Histones can be modified by a range of enzymes, and these modifications can lead to increased or decreased gene transcription [115]. Histone acetylation is an attractive target for intervention as histone deacetylase inhibitors are available and have shown efficacy in a broad range of fibrogenic diseases [116]. Whereas histone modifications are not clearly heritable, DNA methylation is stable and passed on from mother to daughter cells with high fidelity [117].

\section{Summary}

The peritoneal membrane is a fairly simple structure of vital importance to patients who are reliant on PD dialysis as their renal replacement modality. Injury to the peritoneal membrane is a complex process brought about by the dialysis procedure and patient specific factors including genetic predisposition and epigenetic modification. Remarkable strides have been made in understanding the basic mechanisms of peritoneal membrane injury and we hope that these insights will lead to therapeutic interventions that will improve the quality and quantity of life of dialysis patients.

\section{Conflict of Interests}

The authors declare that there is no conflict of interests regarding the publication of this paper.

\section{References}

[1] M. Numata, M. Nakayama, S. Nimura, M. Kawakami, B. Lindholm, and Y. Kawaguchi, "Association between an increased surface area of peritoneal microvessels and a high peritoneal solute transport rate," Peritoneal Dialysis International, vol. 23, no. 2, pp. 116-122, 2003.

[2] M. Rumpsfeld, S. P. McDonald, and D. W. Johnson, "Higher peritoneal transport status is associated with higher mortality and technique failure in the Australian and New Zealand peritoneal dialysis patient populations," Journal of the American Society of Nephrology, vol. 17, no. 1, pp. 271-278, 2006.

[3] K. S. Brimble, M. Walker, P. J. Margetts, K. K. Kundhal, and C. G. Rabbat, "Meta-analysis: peritoneal membrane transport, mortality, and technique failure in peritoneal dialysis," Journal of the American Society of Nephrology, vol. 17, no. 9, pp. 25912598, 2006.

[4] S. H. Chung, O. Heimbürger, and B. Lindholm, "Poor outcomes for fast transporters on PD: The rise and fall of a clinical concern," Seminars in Dialysis, vol. 21, no. 1, pp. 7-10, 2008.

[5] D. Sobiecka, J. Waniewski, A. Weryński, and B. Lindholm, "Peritoneal fluid transport in CAPD patients with different transport rates of small solutes," Peritoneal Dialysis International, vol. 24, no. 3, pp. 240-251, 2004.

[6] A. H. Tzamaloukas, M. C. Saddler, G. H. Murata et al., "Symptomatic fluid retention in patients on continuous peritoneal dialysis," Journal of the American Society of Nephrology, vol. 6, no. 2, pp. 198-206, 1995.

[7] Z. Tonbul, L. Altintepe, Ç. Sözlü, M. Yeksan, A. Yildiz, and S. Türk, "The association of peritoneal transport properties with 24-hour blood pressure levels in CARP patients," Peritoneal Dialysis International, vol. 23, no. 1, pp. 46-52, 2003.

[8] R. N. Foley, P. S. Parfrey, J. D. Harnett, G. M. Kent, D. C. Murray, and P. E. Barre, "Impact of hypertension on cardiomyopathy, 
morbidity and mortality in end-stage renal disease," Kidney International, vol. 49, no. 5, pp. 1379-1385, 1996.

[9] S. Sezer, E. Tutal, Z. Arat et al., "Peritoneal transport status influence on atherosclerosis/inflammation in CAPD patients," Journal of Renal Nutrition, vol. 15, no. 4, pp. 427-434, 2005.

[10] B. Rippe, G. Stelin, and B. Haraldsson, "Computer simulations of peritoneal fluid transport in CAPD," Kidney International, vol. 40, no. 2, pp. 315-325, 1991.

[11] B. Rippe and D. Venturoli, "Simulations of osmotic ultrafiltration failure in CAPD using a serial three-pore membrane/fiber matrix model," The American Journal of Physiology-Renal Physiology, vol. 292, no. 3, pp. F1035-F1043, 2007.

[12] P. J. Margetts, S. Gyorffy, M. Kolb et al., "Antiangiogenic and antifibrotic gene therapy in a chronic infusion model of peritoneal dialysis in rats," Journal of the American Society of Nephrology, vol. 13, no. 3, pp. 721-728, 2002.

[13] P. J. Margetts, M. Kolb, T. Galt, C. M. Hoff, T. R. Shockley, and J. Gauldie, "Gene transfer of transforming growth factor- $\beta 1$ to the rat peritoneum: effects on membrane function," Journal of the American Society of Nephrology, vol. 12, no. 10, pp. 2029-2039, 2001.

[14] P. J. Margetts and D. N. Churchill, "Acquired ultrafiltration dysfunction in peritoneal dialysis patients," Journal of the American Society of Nephrology, vol. 13, no. 11, pp. 2787-2794, 2002.

[15] J. D. Williams, K. J. Craig, N. Topley et al., "Morphologic changes in the peritoneal membrane of patients with renal disease," Journal of the American Society of Nephrology, vol. 13, no. 2, pp. 470-479, 2002.

[16] J. D. Williams, K. J. Craig, N. Topley et al., "Morphologic changes in the peritoneal membrane of patients with renal disease," Journal of the American Society of Nephrology, vol. 13, no. 2, pp. 470-479, 2002.

[17] S. J. Davies, "Longitudinal relationship between solute transport and ultrafiltration capacity in peritoneal dialysis patients," Kidney International, vol. 66, no. 6, pp. 2437-2445, 2004.

[18] G. Garosi and N. di Paolo, "Peritoneal sclerosis: one or two nosological entities," Seminars in Dialysis, vol. 13, no. 5, pp. 297$308,2000$.

[19] J. Rubin, G. A. Herrera, and D. Collins, "An autopsy study of the peritoneal cavity from patients on continuous ambulatory peritoneal dialysis," The American Journal of Kidney Diseases, vol. 18, no. 1, pp. 97-102, 1991.

[20] K. Honda and H. Oda, "Pathology of encapsulating peritoneal sclerosis," Peritoneal Dialysis International, vol. 25, no. 4, pp. S19-S29, 2005.

[21] H. Acloque, M. S. Adams, K. Fishwick, M. Bronner-Fraser, and M. A. Nieto, "Epithelial-mesenchymal transitions: the importance of changing cell state in development and disease," Journal of Clinical Investigation, vol. 119, no. 6, pp. 1438-1449, 2009.

[22] A. Moustakas and C.-H. Heldin, "Signaling networks guiding epithelial-mesenchymal transitions during embryogenesis and cancer progression," Cancer Science, vol. 98, no. 10, pp. 15121520, 2007.

[23] R. Kalluri and R. A. Weinberg, "The basics of epithelialmesenchymal transition," Journal of Clinical Investigation, vol. 119, no. 6, pp. 1420-1428, 2009.

[24] B. Hinz, S. H. Phan, V. J. Thannickal, A. Galli, M.-L. BochatonPiallat, and G. Gabbiani, "The myofibroblast: one function, multiple origins," The American Journal of Pathology, vol. 170, no. 6, pp. 1807-1816, 2007.
[25] F. R. Bob, G. Gluhovschi, D. Herman et al., "Histological, immunohistochemical and biological data in assessing interstitial fibrosis in patients with chronic glomerulonephritis," Acta Histochemica, vol. 110, no. 3, pp. 196-203, 2008.

[26] L. S. Aroeira, J. Loureiro, G. T. González-Mateo et al., "Characterization of epithelial-to-mesenchymal transition of mesothelial cells in a mouse model of chronic peritoneal exposure to high glucose dialysate," Peritoneal Dialysis International, vol. 28, supplement 5, pp. S29-S33, 2008.

[27] E. J. Oh, H. M. Ryu, S. Y. Choi et al., "Impact of low glucose degradation product bicarbonate/lactate-buffered dialysis solution on the epithelial-mesenchymal transition of peritoneum," The American Journal of Nephrology, vol. 31, no. 1, pp. 58-67, 2009.

[28] L. S. Aroeira, A. Aguilera, J. A. Sánchez-Tomero et al., "Epithelial to mesenchymal transition and peritoneal membrane failure in peritoneal dialysis patients: pathologic significance and potential therapeutic interventions," Journal of the American Society of Nephrology, vol. 18, no. 7, pp. 2004-2013, 2007.

[29] P. J. Margetts, P. Bonniaud, L. Liu et al., "Transient overexpression of TGF- $\beta 1$ induces epithelial mesenchymal transition in the rodent peritoneum," Journal of the American Society of Nephrology, vol. 16, no. 2, pp. 425-436, 2005.

[30] M.-A. Yu, K.-S. Shin, J. H. Kim et al., "HGF and BMP-7 ameliorate high glucose-induced epithelial-to-mesenchymal transition of peritoneal mesothelium," Journal of the American Society of Nephrology, vol. 20, no. 3, pp. 567-581, 2009.

[31] P. Patel, Y. Sekiguchi, K.-H. Oh, S. E. Patterson, M. R. J. Kolb, and P. J. Margetts, "Smad3-dependent and-independent pathways are involved in peritoneal membrane injury," Kidney International, vol. 77, no. 4, pp. 319-328, 2010.

[32] P. J. Margetts, "Twist: a new player in the epithelial-mesenchymal transition of the peritoneal mesothelial cells," Nephrology Dialysis Transplantation, vol. 27, no. 11, pp. 3978-3981, 2012.

[33] C. Li, Y. Ren, X. Jia et al., "Twist overexpression promoted epithelial-to-mesenchymal transition of human peritoneal mesothelial cells under high glucose," Nephrology Dialysis Transplantation, vol. 27, no. 11, pp. 4119-4124, 2012.

[34] J. H. Cho, J. Y. Do, E. J. Oh et al., "Are ex vivo mesothelial cells representative of the in vivo transition from epithelialto-mesenchymal cells in peritoneal membrane?" Nephrology Dialysis Transplantation, vol. 27, no. 5, pp. 1768-1779, 2012.

[35] I. Hirahara, Y. Ishibashi, S. Kaname, E. Kusano, and T. Fujita, "Methylglyoxal induces peritoneal thickening by mesenchymallike mesothelial cells in rats," Nephrology Dialysis Transplantation, vol. 24, no. 2, pp. 437-447, 2009.

[36] Y. Liu, "New insights into epithelial-mesenchymal transition in kidney fibrosis," Journal of the American Society of Nephrology, vol. 21, no. 2, pp. 212-222, 2010.

[37] B. D. Humphreys, S.-L. Lin, A. Kobayashi et al., "Fate tracing reveals the pericyte and not epithelial origin of myofibroblasts in kidney fibrosis," American Journal of Pathology, vol. 176, no. 1, pp. 85-97, 2010.

[38] Y. T. Chen, Y. T. Chang, S. Y. Pan et al., "Lineage tracing reveals distinctive fates for mesothelial cells and submesothelial fibroblasts during peritoneal injury," Journal of the American Society of Nephrology. In press.

[39] P. Patel, J. West-Mays, M. Kolb, J.-C. Rodrigues, C. M. Hoff, and P. J. Margetts, "Platelet derived growth factor B and epithelial mesenchymal transition of peritoneal mesothelial cells," Matrix Biology, vol. 29, no. 2, pp. 97-106, 2010. 
[40] Y. Sekiguchi, J. Zhang, S. Patterson et al., "Rapamycin inhibits transforming growth factor $\beta$-induced peritoneal angiogenesis by blocking the secondary hypoxic response," Journal of Cellular and Molecular Medicine, vol. 16, no. 8, pp. 1934-1945, 2012.

[41] J. Zhang, K.-H. Oh, H. Xu, and P. J. Margetts, "Vascular endothelial growth factor expression in peritoneal mesothelial cells undergoing transdifferentiation," Peritoneal Dialysis International, vol. 28, no. 5, pp. 497-504, 2008.

[42] L. S. Aroeira, A. Aguilera, R. Selgas et al., "Mesenchymal conversion of mesothelial cells as a mechanism responsible for high solute transport rate in peritoneal dialysis: role of vascular endothelial growth factor," American Journal of Kidney Diseases, vol. 46, no. 5, pp. 938-948, 2005.

[43] A. B. Roberts, M. B. Sporn, and R. K. Assoian, "Transforming growth factor type $\beta$ : rapid induction of fibrosis and angiogenesis in vivo and stimulation of collagen formation in vitro," Proceedings of the National Academy of Sciences of the United States of America, vol. 83, no. 12, pp. 4167-4171, 1986.

[44] P. J. Margetts, P. Bonniaud, L. Liu et al., "Transient overexpression of TGF- $\beta 1$ induces epithelial mesenchymal transition in the rodent peritoneum," Journal of the American Society of Nephrology, vol. 16, no. 2, pp. 425-436, 2005.

[45] Y. Kyuden, T. Ito, T. Masaki, N. Yorioka, and N. Kohno, "TGF- $\beta 1$ induced by high glucose is controlled by angiotensinconverting enzyme inhibitor and angiotensin II receptor blocker on cultured human peritoneal mesothelial cells," Peritoneal Dialysis International, vol. 25, no. 5, pp. 483-491, 2005.

[46] G. Conti, A. Amore, P. Cirina, L. Peruzzi, S. Balegno, and R. Coppo, "Glycated adducts induce mesothelial cell transdifferentiation: role of glucose and icodextrin dialysis solutions," Journal of Nephrology, vol. 21, no. 3, pp. 426-437, 2008.

[47] P. J. Margetts, M. Kolb, L. Yu et al., "Inflammatory cytokines, angiogenesis, and fibrosis in the rat peritoneum," The American Journal of Pathology, vol. 160, no. 6, pp. 2285-2294, 2002.

[48] Y. Sun, F. Zhu, X. Yu et al., “Treatment of established peritoneal fibrosis by gene transfer of Smad7 in a rat model of peritoneal dialysis," American Journal of Nephrology, vol. 30, no. 1, pp. 8494, 2009.

[49] H. Guo, J. C. K. Leung, M. F. Lam et al., "Smad7 transgene attenuates peritoneal fibrosis in uremic rats treated with peritoneal dialysis," Journal of the American Society of Nephrology, vol. 18, no. 10, pp. 2689-2703, 2007.

[50] J. Loureiro, M. Schilte, A. Aguilera et al., "BMP-7 blocks mesenchymal conversion of mesothelial cells and prevents peritoneal damage induced by dialysis fluid exposure," Nephrology Dialysis Transplantation, vol. 25, no. 4, pp. 1098-1108, 2010.

[51] A. S. Gangji, K. S. Brimble, and P. J. Margetts, "Association between markers of inflammation, fibrosis and hypervolemia in peritoneal dialysis patients," Blood Purification, vol. 28, no. 4, pp. 354-358, 2009.

[52] J.-H. Cho, I.-K. Hur, C.-D. Kim et al., "Impact of systemic and local peritoneal inflammation on peritoneal solute transport rate in new peritoneal dialysis patients: a 1-year prospective study," Nephrology Dialysis Transplantation, vol. 25, no. 6, pp. 1964-1973, 2010.

[53] L. Liu, C.-X. Shi, A. Ghayur et al., "Prolonged peritoneal gene expression using a helper-dependent adenovirus," Peritoneal Dialysis International, vol. 29, no. 5, pp. 508-516, 2009.

[54] W. Peng, Q. Zhou, X. Ao, R. Tang, and Z. Xiao, "Inhibition of Rho-kinase alleviates peritoneal fibrosis and angiogenesis in a rat model of peritoneal dialysis," Renal Failure, vol. 35, no. 7, pp. 958-966, 2013.
[55] R. Strippoli, I. Benedicto, M. L. Perez Lozano et al., "Inhibition of transforming growth factor-activated kinase 1 (TAK1) blocks and reverses epithelial to mesenchymal transition of mesothelial cells," PLoS ONE, vol. 7, no. 2, Article ID e31492, 2012.

[56] S. Kokubo, N. Sakai, K. Furuichi et al., "Activation of p38 mitogen-activated protein kinase promotes peritoneal fibrosis by regulating fibrocytes," Peritoneal Dialysis International, vol. 32, no. 1, pp. 10-19, 2012.

[57] P. J. Margetts, M. Kolb, L. Yu, C. M. Hoff, and J. Gauldie, "A chronic inflammatory infusion model of peritoneal dialysis in rats," Peritoneal Dialysis International, vol. 21, no. 3, pp. S368S372, 2001.

[58] J. Plum, S. Hermann, A. Fusshöller et al., "Peritoneal sclerosis in peritoneal dialysis patients related to dialysis settings and peritoneal transport properties," Kidney International, Supplement, vol. 59, no. 78, pp. S42-S47, 2001.

[59] M. A. M. Mateijsen, A. C. van der Wal, P. M. E. M. Hendriks et al., "Vascular and interstitial changes in the peritoneum of CAPD patients with peritoneal sclerosis," Peritoneal Dialysis International, vol. 19, no. 6, pp. 517-525, 1999.

[60] M. C. Dickson, J. S. Martin, F. M. Cousins, A. B. Kulkarni, S. Karlsson, and R. J. Akhurst, "Defective haematopoiesis and vasculogenesis in transforming growth factor- $\beta 1$ knock out mice," Development, vol. 121, no. 6, pp. 1845-1854, 1995.

[61] T. O. Daniel and D. Abrahamson, "Endothelial signal integration in vascular assembly," Annual Review of Physiology, vol. 62, pp. 649-671, 2000.

[62] S. P. Oh, T. Seki, K. A. Goss et al., "Activin receptor-like kinase 1 modulates transforming growth factor- $\beta 1$ signaling in the regulation of angiogenesis," Proceedings of the National Academy of Sciences of the United States of America, vol. 97, no. 6, pp. 26262631, 2000.

[63] J. Folkman and P. A. D'Amore, "Blood vessel formation: what is its molecular basis?” Cell, vol. 87, no. 7, pp. 1153-1155, 1996.

[64] T. Sánchez-Elsner, L. M. Botella, B. Velasco, A. Corbí, L. Attisano, and C. Bernabéu, "Synergistic cooperation between hypoxia and transforming growth factor-beta pathways on human vascular endothelial growth factor gene expression," The Journal of Biological Chemistry, vol. 276, no. 42, pp. 3852738535, 2001.

[65] G. Breier, S. Blum, J. Peli et al., "Transforming growth factor$\beta$ and RAS regulate the VEGF/VEGF-receptor system during tumor angiogenesis," International Journal of Cancer, vol. 97, no. 2, pp. 142-148, 2002.

[66] M. C. Iglesias-de la Cruz, F. N. Ziyadeh, M. Isono et al., "Effects of high glucose and TGF- $\beta 1$ on the expression of collagen IV and vascular endothelial growth factor in mouse podocytes," Kidney International, vol. 62, no. 3, pp. 901-913, 2002.

[67] B. B. Scott, P. F. Zaratin, A. Colombo, M. J. Hansbury, J. D. Winkler, and J. R. Jackson, "Constitutive expression of angiopoietin- 1 and -2 and modulation of their expression by inflammatory cytokines in rheumatoid arthritis synovial fibroblasts," The Journal of Rheumatology, vol. 29, no. 2, pp. 230-239, 2002.

[68] P. J. Margetts, M. Kolb, C. M. Hoff, and J. Gauldie, “The role of angiopoietins in resolution of angiogenesis resulting from adenovirual mediated gene transfer of TGF $\beta 1$ or VEGF to the rat peritoneum," Journal of the American Society of Nephrology, vol. 12, Abstract 433A, no. 10, 2001.

[69] V. E. Belozerov and E. G. van Meir, "Hypoxia inducible factor-1: a novel target for cancer therapy," Anti-Cancer Drugs, vol. 16, no. 9, pp. 901-909, 2005. 
[70] P. K. Majumder, P. G. Febbo, R. Bikoff et al., "mTOR inhibition reverses Akt-dependent prostate intraepithelial neoplasia through regulation of apoptotic and HIF-1-dependent pathways," Nature Medicine, vol. 10, no. 6, pp. 594-601, 2004.

[71] H. O. Akman, H. Zhang, M. A. Q. Siddiqui, W. Solomon, E. L. P. Smith, and O. A. Batuman, "Response to hypoxia involves transforming growth factor- $\beta 2$ and Smad proteins in human endothelial cells," Blood, vol. 98, no. 12, pp. 3324-3331, 2001.

[72] H. Zhang, H. O. Akman, E. L. P. Smith et al., "Cellular response to hypoxia involves signaling via Smad proteins," Blood, vol. 101, no. 6, pp. 2253-2260, 2003.

[73] E. Papakonstantinou, A. J. Aletras, M. Roth, M. Tamm, and G. Karakiulakis, "Hypoxia modulates the effects of transforming growth factor- $\beta$ isoforms on matrix-formation by primary human lung fibroblasts," Cytokine, vol. 24, no. 1-2, pp. 25-35, 2003.

[74] M. Kolb, P. Bonniaud, T. Galt et al., "Differences in the fibrogenic response after transfer of active transforming growth factor- $\beta 1$ gene to lungs of "fibrosis-prone" and "fibrosisresistant" mouse strains," American Journal of Respiratory Cell and Molecular Biology, vol. 27, no. 2, pp. 141-150, 2002.

[75] P. Bonniaud, P. J. Margetts, M. Kolb et al., "Adenoviral gene transfer of connective tissue growth factor in the lung induces transient fibrosis," The American Journal of Respiratory and Critical Care Medicine, vol. 168, no. 7, pp. 770-778, 2003.

[76] D. F. Higgins, M. P. Biju, Y. Akai, A. Wutz, R. S. Johnson, and V. H. Haase, "Hypoxic induction of Ctgf is directly mediated by Hif-1," The American Journal of Physiology-Renal Physiology, vol. 287, no. 6, pp. F1223-F1232, 2004.

[77] D. F. Higgins, K. Kimura, W. M. Bernhardt et al., "Hypoxia promotes fibrogenesis in vivo via HIF-1 stimulation of epithelial-tomesenchymal transition," Journal of Clinical Investigation, vol. 117, no. 12, pp. 3810-3820, 2007.

[78] K. Chaudhary and R. Khanna, "Biocompatible peritoneal dialysis solutions: do we have one?" Clinical Journal of the American Society of Nephrology, vol. 5, no. 4, pp. 723-732, 2010.

[79] P. G. Blake, A. K. Jain, and S. Yohanna, "Biocompatible peritoneal dialysis solutions: many questions but few answers," Kidney International, vol. 84, no. 5, pp. 864-866, 2013.

[80] D.-H. Kang, Y.-S. Hong, H. J. Lim, J.-H. Choi, D.-S. Han, and K.L. Yoon, "High glucose solution and spent dialysate stimulate the synthesis of transforming growth factor- $\beta 1$ of human peritoneal mesothelial cells: effect of cytokine costimulation," Peritoneal Dialysis International, vol. 19, no. 3, pp. 221-230, 1999.

[81] S. J. Davies, L. Phillips, P. F. Naish, and G. I. Russell, "Peritoneal glucose exposure and changes in membrane solute transport with time on peritoneal dialysis," Journal of the American Society of Nephrology, vol. 12, no. 5, pp. 1046-1051, 2001.

[82] Y. Wen, Q. Guo, X. Yang et al., "High glucose concentrations in peritoneal dialysis are associated with all-cause and cardiovascular disease mortality in continuous ambulatory peritoneal dialysis patients," Peritoneal Dialysis International, 2013.

[83] A. S. de Vriese, R. G. Tilton, C. C. Stephan, and N. H. Lameire, "Vascular endothelial growth factor is essential for hyperglycemia-induced structural and functional alterations of the peritoneal membrane," Journal of the American Society of Nephrology, vol. 12, no. 8, pp. 1734-1741, 2001.

[84] G. Clerbaux, J. Francart, P. Wallemacq, A. Robert, and E. Goffin, "Evaluation of peritoneal transport properties at onset of peritoneal dialysis and longitudinal follow-up," Nephrology Dialysis Transplantation, vol. 21, no. 4, pp. 1032-1039, 2006.
[85] A. S. Rodrigues, M. Martins, J. C. Korevaar et al., "Evaluation of peritoneal transport and membrane status in peritoneal dialysis: focus on incident fast transporters," The American Journal of Nephrology, vol. 27, no. 1, pp. 84-91, 2007.

[86] A. S. de Vriese, A. Flyvbjerg, S. Mortier, R. G. Tilton, and N. H. Lameire, "Inhibition of the interaction of age-rage prevents hyperglycemia-induced fibrosis of the peritoneal membrane," Journal of the American Society of Nephrology, vol. 14, no. 8, pp. 2109-2118, 2003.

[87] Y. Cho, D. W. Johnson, J. C. Craig et al., "Biocompatible dialysis fluids for peritoneal dialysis," Cochrane Database of Systematic Reviews, vol. 3, Article ID CD007554, 2014.

[88] D. W. Johnson, F. G. Brown, M. Clarke et al., "The effect of low glucose degradation product, neutral $\mathrm{pH}$ versus standard peritoneal dialysis solutions on peritoneal membrane function: the balANZ trial," Nephrology Dialysis Transplantation, vol. 27, no. 12, pp. 4445-4453, 2012.

[89] C.-Y. Lin, W.-P. Chen, L.-Y. Yang, A. Chen, and T.-P. Huang, "Persistent transforming growth factor-beta 1 expression may predict peritoneal fibrosis in CAPD patients with frequent peritonitis occurrence," American Journal of Nephrology, vol. 18, no. 6, pp. 513-519, 1998.

[90] P. J. Margetts, M. Kolb, L. Yu et al., "Inflammatory cytokines, angiogenesis, and fibrosis in the rat peritoneum," American Journal of Pathology, vol. 160, no. 6, pp. 2285-2294, 2002.

[91] G. del Peso, M. J. Fernández-Reyes, C. Hevia et al., "Factors influencing peritoneal transport parameters during the first year on peritoneal dialysis: peritonitis is the main factor," Nephrology Dialysis Transplantation, vol. 20, pp. 1201-1206, 2005.

[92] A. T. van Diepen, E. S. Van, D. G. Struijk, and R. T. Krediet, "The first peritonitis episode alters the natural course of peritoneal membrane characteristics in peritoneal dialysis patients," Peritoneal Dialysis International, 2014.

[93] T. Hirano, K. Yasukawa, H. Harada et al., "Complementary DNA for a novel human interleukin (BSF-2) that induces B lymphocytes to produce immunoglobulin," Nature, vol. 324, no. 6092, pp. 73-76, 1986.

[94] K.-H. Oh, J. Y. Jung, M. O. Yoon et al., "Intra-peritoneal interleukin-6 system is a potent determinant of the baseline peritoneal solute transport in incident peritoneal dialysis patients," Nephrology Dialysis Transplantation, vol. 25, no. 5, pp. 1639$1646,2010$.

[95] M. Lambie, J. Chess, K. L. Donovan et al., "Independent effects of systemic and peritoneal inflammation on peritoneal dialysis survival," Journal of the American Society of Nephrology, vol. 24, no. 12, pp. 2071-2080, 2013.

[96] S. Combet, M.-L. Ferrier, M. van Landschoot et al., "Chronic uremia induces permeability changes, increased nitric oxide synthase expression, and structural modifications in the peritoneum," Journal of the American Society of Nephrology, vol. 12, no. 10 , pp. $2146-2157,2001$.

[97] K. Honda, C. Hamada, M. Nakayama et al., "Impact of uremia, diabetes, and peritoneal dialysis itself on the pathogenesis of peritoneal sclerosis: a quantitative study of peritoneal membrane morphology," Clinical Journal of the American Society of Nephrology, vol. 3, no. 3, pp. 720-728, 2008.

[98] G. Gillerot, E. Goffin, C. Michel et al., "Genetic and clinical factors influence the baseline permeability of the peritoneal membrane," Kidney International, vol. 67, no. 6, pp. 2477-2487, 2005. 
[99] Y.-H. Hwang, M.-J. Son, J. Yang et al., "Effects of interleukin6 T15A single nucleotide polymorphism on baseline peritoneal solute transport rate in incident peritoneal dialysis patients," Peritoneal Dialysis International, vol. 29, no. 1, pp. 81-88, 2009.

[100] T. Y.-H. Wong, C.-C. Szeto, C. Y.-K. Szeto, K.-B. Lai, K.-M. Chow, and P. K.-T. Li, "Association of ENOS polymorphism with basal peritoneal membrane function in uremic patients," The American Journal of Kidney Diseases, vol. 42, no. 4, pp. 781-786, 2003.

[101] A. Akcay, H. Micozkadioglu, F. B. Atac, E. Agca, and F. N. Ozdemir, "Relationship of ENOS and RAS gene polymorphisms to initial peritoneal transport status in peritoneal dialysis patients," Nephron-Clinical Practice, vol. 104, no. 1, pp. c41-c46, 2006.

[102] Y. Maruyama, M. Numata, M. Nakayama et al., "Relationship between the -374T/A receptor of advanced glycation end products gene polymorphism and peritoneal solute transport status at the initiation of peritoneal dialysis," Therapeutic Apheresis and Dialysis, vol. 11, no. 4, pp. 301-305, 2007.

[103] C.-C. Szeto, K.-M. Chow, P. Poon, C. Y.-K. Szeto, T. Y.-H. Wong, and P. K.-T. Li, "Genetic polymorphism of VEGF: impact on longitudinal change of peritoneal transport and survival of peritoneal dialysis patients," Kidney International, vol. 65, no. 5, pp. 1947-1955, 2004.

[104] Y.-T. Lee, Y.-C. Tsai, Y.-K. Yang et al., "Association between interleukin-10 gene polymorphism $-592(\mathrm{~A} / \mathrm{C})$ and peritoneal transport in patients undergoing peritoneal dialysis," Nephrology, vol. 16, no. 7, pp. 663-671, 2011.

[105] M. Numata, M. Nakayama, T. Hosoya et al., "Possible pathologic involvement of receptor for advanced glycation end products (RAGE) for development of encapsulating peritoneal sclerosis in Japanese CAPD patients," Clinical Nephrology, vol. 62, no. 6, pp. $455-460,2004$.

[106] P. J. Margetts, C. Hoff, L. Liu et al., "Transforming growth factor $\beta$-induced peritoneal fibrosis is mouse strain dependent," Nephrology Dialysis Transplantation, vol. 28, no. 8, pp. 20152027, 2013.

[107] A. Leelahavanichkul, Q. Yan, X. Hu et al., "Angiotensin II overcomes strain-dependent resistance of rapid CKD progression in a new remnant kidney mouse model," Kidney International, vol. 78, no. 11, pp. 1136-1153, 2010.

[108] S. Hillebrandt, C. Goos, S. Matern, and F. Lammert, "Genomewide analysis of hepatic fibrosis in inbred mice identifies the susceptibility locus Hfibl on chromosome 15," Gastroenterology, vol. 123, no. 6, pp. 2041-2051, 2002.

[109] S. W. M. Van Den Borne, V. A. M. Van De Schans, A. E. Strzelecka et al., "Mouse strain determines the outcome of wound healing after myocardial infarction," Cardiovascular Research, vol. 84, no. 2, pp. 273-282, 2009.

[110] P. W. Noble, C. E. Barkauskas, and D. Jiang, "Pulmonary fibrosis: patterns and perpetrators," Journal of Clinical Investigation, vol. 122, no. 8, pp. 2756-2762, 2012.

[111] E. M. Zeisberg and M. Zeisberg, "The role of promoter hypermethylation in fibroblast activation and fibrogenesis," The Journal of Pathology, vol. 229, no. 2, pp. 264-273, 2013.

[112] G. Balasubramaniam, E. A. Brown, A. Davenport et al., "The Pan-Thames EPS study: treatment and outcomes of encapsulating peritoneal sclerosis," Nephrology Dialysis Transplantation, vol. 24, no. 10, pp. 3209-3215, 2009.

[113] W. Bechtel, S. McGoohan, E. M. Zeisberg et al., "Methylation determines fibroblast activation and fibrogenesis in the kidney," Nature Medicine, vol. 16, no. 5, pp. 544-550, 2010.
[114] J. Mann and D. A. Mann, "Epigenetic regulation of wound healing and fibrosis," Current Opinion in Rheumatology, vol. 25, no. 1, pp. 101-107, 2013.

[115] M. Pang and S. Zhuang, "Histone deacetylase: a potential therapeutic target for fibrotic disorders," Journal of Pharmacology and Experimental Therapeutics, vol. 335, no. 2, pp. 266-272, 2010.

[116] K. van Beneden, I. Mannaerts, M. Pauwels, C. van den Branden, and L. A. van Grunsven, "HDAC inhibitors in experimental liver and kidney fibrosis," Fibrogenesis and Tissue Repair, vol. 6, article 1, 2013.

[117] C. Huang, M. Xu, and B. Zhu, "Epigenetic inheritance mediated by histone lysine methylation: maintaining transcriptional states without the precise restoration of marks?" Philosophical Transactions of the Royal Society B: Biological Sciences, vol. 368, no. 1609, Article ID 20110332, 2013. 


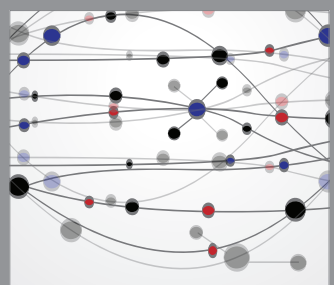

The Scientific World Journal
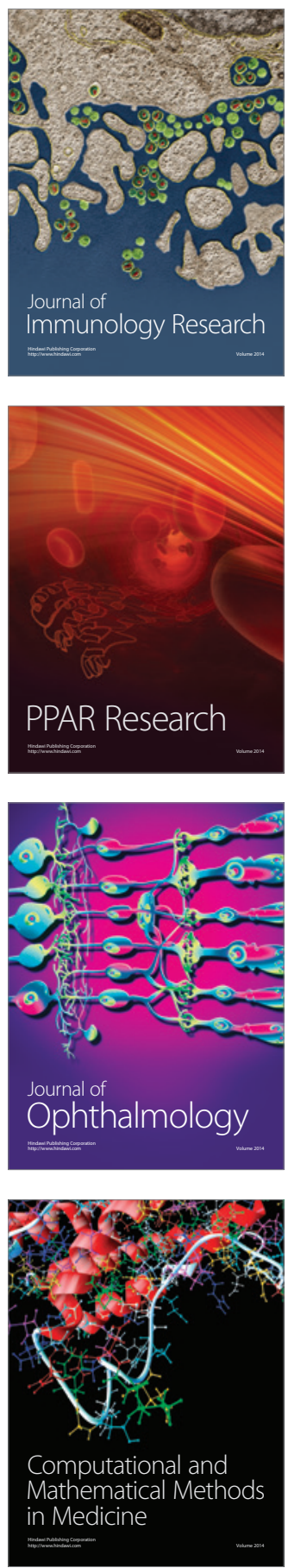

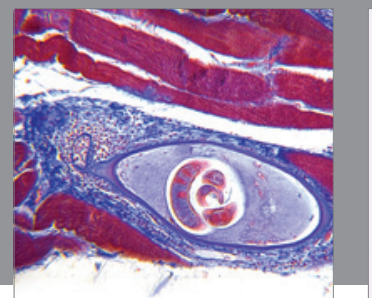

Gastroenterology

Research and Practice
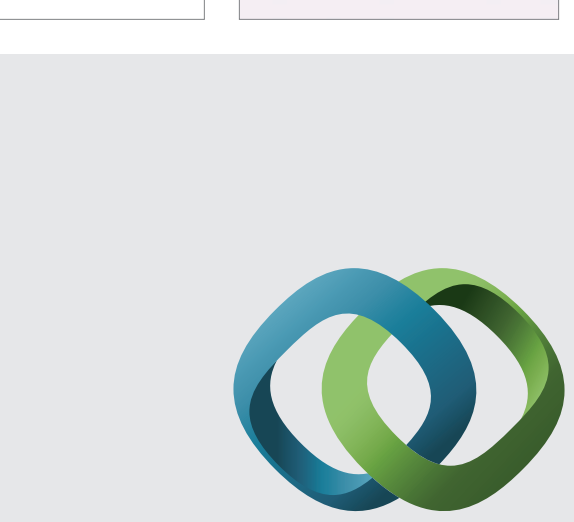

\section{Hindawi}

Submit your manuscripts at

http://www.hindawi.com
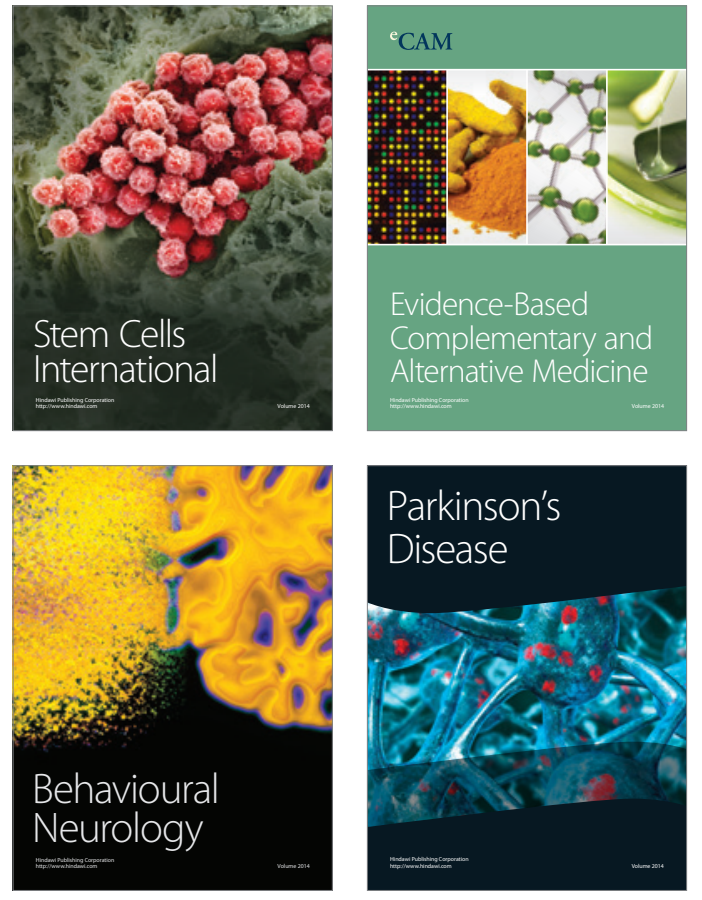
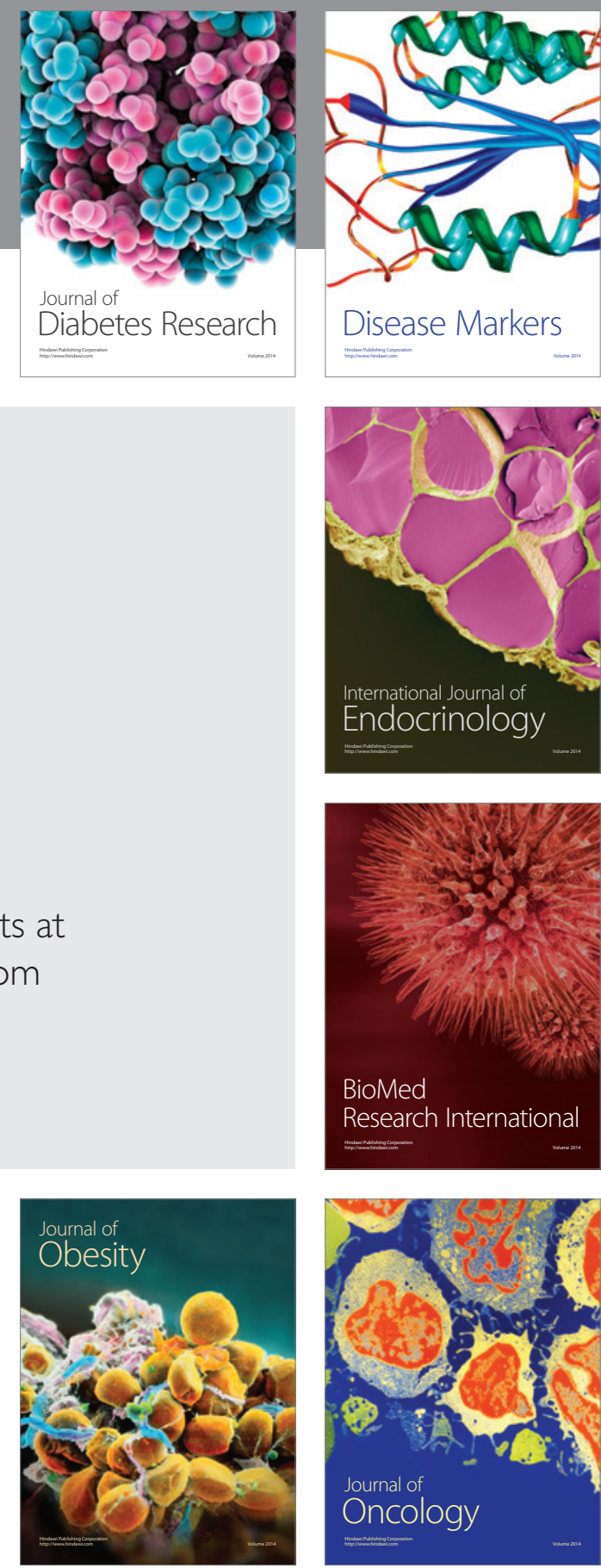

Disease Markers
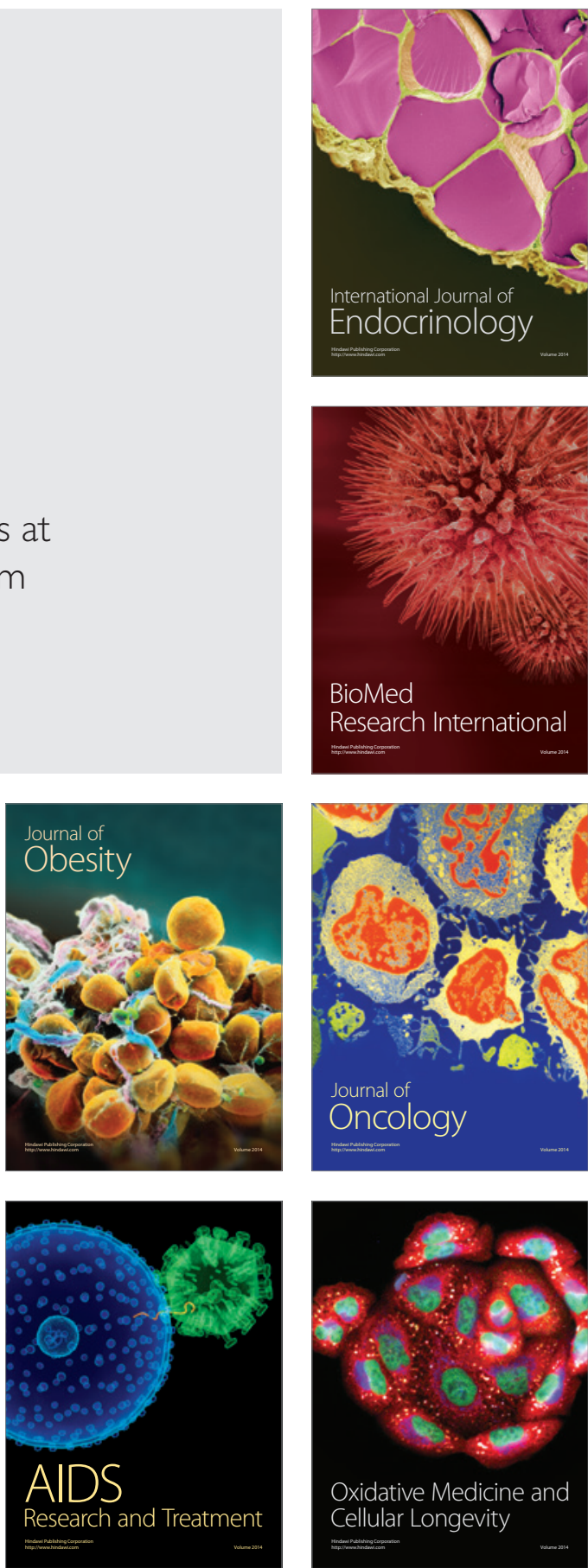